\title{
Using ac dipoles to localize sources of beam coupling impedance
}

\author{
N. Biancacci and R. Tomás \\ CERN, 1211 Geneva, Switzerland \\ (Received 14 January 2015; published 3 May 2016)
}

\begin{abstract}
The beam coupling impedance is one of the main sources of beam instabilities and emittance blow up in circular accelerators. A refined machine impedance evaluation is therefore required in order to understand and model intensity dependent effects and instabilities that may limit the machine performance. For this reason, many impedance source localization techniques have been developed. In this work we present the impedance localization technique based on the observation of phase advance versus intensity at the beam position monitors using ac dipoles to force betatron oscillations. We present analytical formulas for the interpretation of measurements together with simulations to benchmark and illustrate the equations. Studies on the method accuracy for different Fourier transform algorithms are presented as well as first exploratory measurements performed in the LHC.
\end{abstract}

DOI: 10.1103/PhysRevAccelBeams.19.054001

\section{INTRODUCTION}

Elements like the resistive wall of the beam pipe, $\mathrm{rf}$ cavities, kickers and collimators are often large impedance sources. Measuring the betatron coherent frequency shift with intensity gives information on the total reactive transverse impedance according to Sacherer's theory [1]. An extension of this method for measuring the reactive part of transverse localized impedances was proposed the first time in 1995 at CERN [2] by measuring the impedanceinduced betatron phase advance shift with intensity. The Large Electron-Positron collider (LEP) rf sections were found to be important impedance contributors.

A method, based on the impedance-induced orbit shift with intensity, was proposed in 1999 in the Novosibirsk VEPP-4M electron-positron storage ring [3] and in 2001 in the Argonne APS synchrotron accelerator [4]. Later in 2002, the same method was tried in the European Synchrotron Radiation Facility (ESRF) [5].

The impedance localization method using phase advance shift with intensity was continued in 2004 in the super proton synchrotron (SPS) [6,7] and in the Brookhaven National Laboratory's Relativistic Heavy Ion Collider (BNL RHIC) [8]. The impact of noise on the measurement and the techniques for reconstructing the impedance location were recently studied in [9].

We briefly review the impedance localization method based on the impedance-induced betatron phase advance shift with intensity. A circulating beam performs betatron oscillations that may be enhanced by means of a kick. The natural transverse coherent modes of oscillation described in

Published by the American Physical Society under the terms of the Creative Commons Attribution 3.0 License. Further distribution of this work must maintain attribution to the author(s) and the published article's title, journal citation, and DOI.
Sacherer's theory are subject to an intensity dependent shift. In the vertical plane for example, the tune $Q_{y}$ shifts with the intensity from the unperturbed tune $Q_{y}^{0}$, according to

$$
Q_{y}-Q_{y}^{0}=-\frac{q^{2} N_{b}}{8 \pi^{3 / 2} \beta^{2} E_{o} \sigma_{\tau}} \oint_{C} \beta_{y}^{0}(s) \operatorname{Im}\left(Z_{y}^{\mathrm{eff}}(s)\right) \mathrm{d} s,
$$

where we consider a Gaussian bunch of longitudinal rms bunch length $\sigma_{\tau}$ in seconds, bunch population $N_{b}$, particle charge $q$, circulating at velocity $\beta c$ with $c$ the speed of light at the energy $E_{o}$. The contribution of an impedance source to the tune shift increases proportionally to the machine unperturbed $\beta_{y}^{0}(s)$ function and the total effective imaginary part of the impedance $Z_{y}^{\text {eff }}(s)$. The integration accounts both for distributed elements, like the beam pipe, and localized impedance sources, like kickers and collimators. For an impedance source localized at $s=s_{k}$ along the accelerator circumference, we can write

$$
Z_{y}^{\mathrm{eff}}(s) \simeq Z_{y_{k}}^{\mathrm{eff}} L_{k} \delta\left(s-s_{k}\right),
$$

where $L_{k}$ accounts for the element length and $\delta(s)$ is the Dirac function. The tune derivative versus intensity $\mathrm{d} Q_{y_{k}} / \mathrm{d} N_{b}$, is therefore

$$
\frac{\mathrm{d} Q_{y_{k}}}{\mathrm{~d} N_{b}}=-\frac{q^{2}}{8 \pi^{3 / 2} \beta^{2} E_{o} \sigma_{\tau}} \beta_{y}^{0}\left(s_{k}\right) \operatorname{Im}\left(Z_{y_{k}}^{\mathrm{eff}}\right) L_{k} .
$$

Considering many impedance sources we have

$$
\frac{\mathrm{d} Q_{y_{\text {tot }}}}{\mathrm{d} N_{b}}=\sum_{k} \frac{\mathrm{d} Q_{y_{k}}}{\mathrm{~d} N_{b}}
$$

Since the tune linearly shifts with intensity we can compare it with the one provoked by an intensity dependent quadrupole error $\mathrm{d} K_{y_{k}} / \mathrm{d} N_{b}$ at the location $s=s_{k}$ 


$$
\frac{\mathrm{d} Q_{y_{k}}}{\mathrm{~d} N_{b}}=\frac{\beta_{y}^{0}\left(s_{k}\right)}{4 \pi} \frac{\mathrm{d} K_{y_{k}}}{\mathrm{~d} N_{b}} L_{k} .
$$

A localized impedance source $Z_{y_{k}}^{\text {eff }}$ will appear as a quadrupolar kick of value

$$
\frac{\mathrm{d} K_{y_{k}}}{\mathrm{~d} N_{b}}=-\frac{q^{2}}{2 \sqrt{\pi} \beta^{2} E_{o} \sigma_{\tau}} \operatorname{Im}\left(Z_{y_{k}}^{\mathrm{eff}}\right) .
$$

Associated with a quadrupolar kick there are also a $\beta$-beating and a phase advance beating wave along the machine. Given a reference beam position monitor (BPM) placed for convention at $s=0$ in the machine, we can define $\mu_{y}(s)$ as the phase advance from the reference BPM position to the $s$ position in units of $2 \pi$. Defining the unperturbed phase advance as $\mu_{y}^{0}(s)$, the phase advance slope versus intensity $\mathrm{d} \mu_{y}(s) / \mathrm{d} N_{b}$ due to an intensity dependent quadrupole error located at $s=s_{k}$ can be calculated as $[9,10]$

$$
\frac{\mathrm{d} \mu_{y}(s)}{\mathrm{d} N_{b}}=\frac{\mathrm{d} Q_{y_{k}}}{\mathrm{~d} N_{b}}\left\{\begin{array}{l}
1+\frac{c(s)^{+} s(s)^{+}}{\sin \left(2 \pi Q_{y}^{0}\right)}, \quad \text { for } s \geq s_{k}, \\
\frac{s(s)^{-} c(s)^{-}}{\sin \left(2 \pi Q_{y}^{0}\right)}, \quad \text { for } s<s_{k},
\end{array}\right.
$$

with

$$
\begin{aligned}
& s(s)^{-}=\sin \left[2 \pi \mu_{y}^{0}(s)\right], \\
& c(s)^{+}=\cos \left[4 \pi \mu_{y}^{0}\left(s_{k}\right)-2 \pi \mu_{y}^{0}(s)\right], \\
& s(s)^{+}=\sin \left[2 \pi \mu_{y}^{0}(s)-2 \pi Q_{y}^{0}\right], \\
& c(s)^{-}=\cos \left[2 \pi \mu_{y}^{0}(s)-4 \pi \mu_{y}^{0}\left(s_{k}\right)+2 \pi Q_{y}^{0}\right] .
\end{aligned}
$$

We notice that $\mathrm{d} \mu_{y}(s) / \mathrm{d} N_{b}$ exhibits a step of $\mathrm{d} Q_{y_{k}} / \mathrm{d} N_{b}$ at $s=s_{k}$. Since the tune shifts associated to impedance are usually negative, the step will be also negative. Measuring the variation of phase advance versus intensity along the machine can be used to infer the impedance locations in the machine.

We conclude noticing that a resistive wall impedance could be treated (on average) as a smoothly distributed quadrupole error provoking a smooth negative slope in the $\mathrm{d} \mu_{y}(s) / \mathrm{d} N_{b}$ curve [9].

\section{AC DIPOLE DRIVEN OSCILLATIONS}

An ac dipole is a radio frequency dipole that produces an oscillating field that excites driven oscillations in the beam. While a normal kick would naturally excite the coherent tune oscillation and sidebands, with an ac dipole it is possible to drive the beam oscillation at different frequencies and maintain coherent oscillations for many turns improving the quality and reproducibility of the optics measurement.

The ac dipole was first introduced and studied in the BNL AGS [11]. Analytical studies on the linear and nonlinear particle motion in the presence of an ac dipole were done in $[12,13]$. Measurements of linear machine optics using ac dipoles were done in Tevatron [10,14],

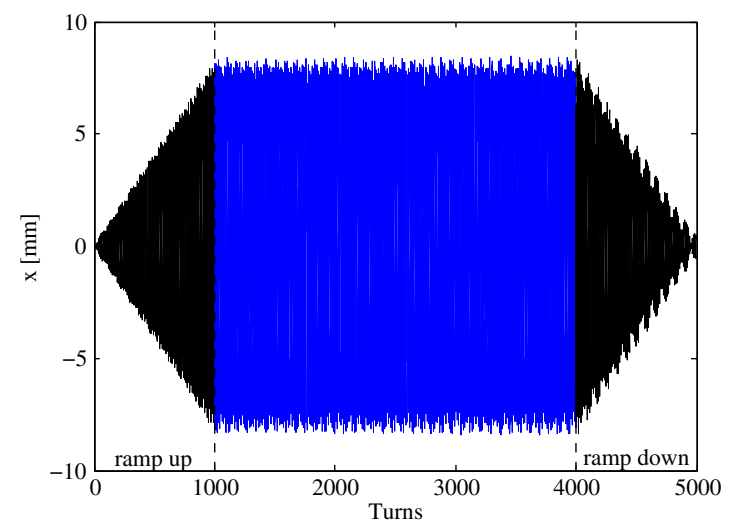

FIG. 1. Typical excitation pattern of a driven oscillation at frequency $Q_{d}$ as simulated in HEADTAIL.

RHIC [15,16] and in the LHC [17] while nonlinear optics measurements were done, for example, in the SPS [18], RHIC [19] and the LHC [20,21].

Figure 1 shows the typical excitation pattern of a driven oscillation at frequency $Q_{d}$ simulated in HEADTAIL $[22,23]$ : the ramps before and after the excitation plateau allow for negligible emittance growth [24]. Figure 2 shows the driven $Q_{d}$ and the natural $Q_{\text {nat }}$ frequencies in the spectrum of the simulated transverse beam oscillation during the ac dipole plateau. As we can see the signal corresponding to the driven frequency is much stronger than the natural tune.

The parameter $\delta=Q_{d}-Q_{\text {nat }}$ is the distance between natural and driven frequencies. The phase advance from the forced ac dipole oscillation deviates from the natural phase advance according to the relation [14]

$$
\tan \left(2 \pi \mu_{d}-\pi Q_{d}\right)=\frac{1+\lambda_{d}}{1-\lambda_{d}} \tan \left(2 \pi \mu_{\mathrm{nat}}-\pi Q_{\mathrm{nat}}\right),
$$

where $\mu_{d}$ is the measured phase advance from the ac dipole location at the driven frequency, $\mu_{\text {nat }}$ is the phase advance at the natural frequency, and $\lambda_{d}$ is given by

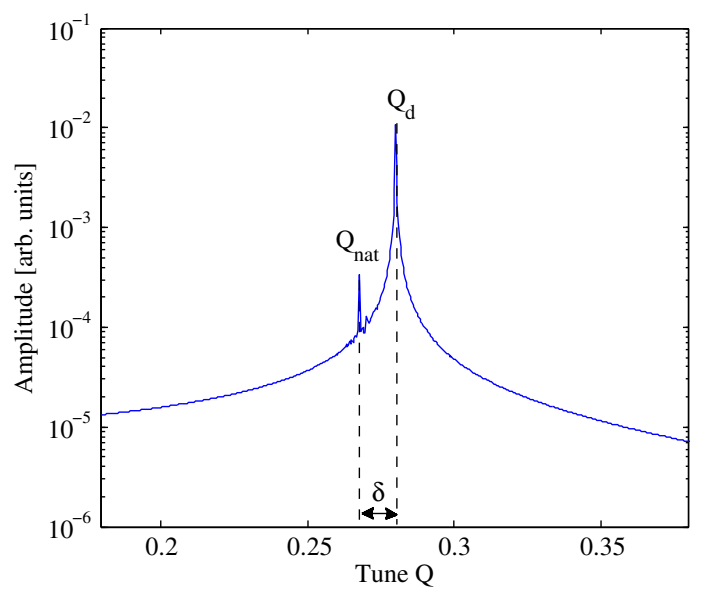

FIG. 2. Simulated spectrum of the transverse beam signal on the ac dipole plateau showing driven $Q_{d}$ and natural $Q_{\text {nat }}$ tunes. 


$$
\lambda_{d}=\frac{\sin \left[\pi\left(Q_{d}-Q_{\text {nat }}\right)\right]}{\sin \left[\pi\left(Q_{d}+Q_{\text {nat }}\right)\right]} .
$$

Analogously, the optics at the driven frequency, can be modeled as the natural optics including a quadrupole error of strength $K_{\mathrm{ac}}$ at the ac dipole location. The strength $K_{\mathrm{ac}}$ is given by [14]

$$
K_{\mathrm{ac}}=2 \frac{\cos \left(2 \pi Q_{\mathrm{nat}}\right)-\cos \left(2 \pi Q_{d}\right)}{\beta_{\mathrm{ac}} \sin \left(2 \pi Q_{\mathrm{nat}}\right)}
$$

with $\beta_{\text {ac }}$ being the beta function at the ac dipole location.

\section{IMPEDANCE LOCALIZATION MEASUREMENTS WITH AC DIPOLE}

We define $Q_{\text {nat }}^{0}$ as the natural tune corresponding to an impedance-free machine (i.e., for virtually $N_{b}=0 \mathrm{ppb}$ ). From Eq. (10) the ac dipole driven optics is given by the natural optics perturbed with the quadrupole error

$$
K_{\mathrm{ac}}^{0}=2 \frac{\cos \left(2 \pi Q_{\mathrm{nat}}^{0}\right)-\cos \left(2 \pi Q_{d}\right)}{\beta_{\mathrm{ac}} \sin \left(2 \pi Q_{\mathrm{nat}}^{0}\right)} .
$$

Increasing the intensity by $\mathrm{d} N_{b}$ a linear tune shift with intensity would modify the coherent natural tune as

$$
Q_{\text {nat }}=Q_{\text {nat }}^{0}+\mathrm{d} Q_{\text {nat }} .
$$

Inserting this information in Eq. (10) to obtain the equivalent ac dipole quadrupole error, we have

$$
K_{\mathrm{ac}}=2 \frac{\cos \left[2 \pi\left(Q_{\mathrm{nat}}^{0}+\mathrm{d} Q_{\mathrm{nat}}\right)\right]-\cos \left(2 \pi Q_{d}\right)}{\beta_{\mathrm{ac}} \sin \left[2 \pi\left(Q_{\mathrm{nat}}^{0}+\mathrm{d} Q_{\mathrm{nat}}\right)\right]} .
$$

Developing at first order considering small tune shifts $\mathrm{d} Q_{\text {nat }}$ we get

$$
K_{\mathrm{ac}} \simeq K_{\mathrm{ac}}^{0}-\frac{4 \pi}{\beta_{a c}} \mathrm{~d} Q_{\mathrm{nat}}\left[1+\frac{\beta_{\mathrm{ac}}}{2} \frac{K_{\mathrm{ac}}^{0}}{\tan \left(2 \pi Q_{\mathrm{nat}}^{0}\right)}\right] .
$$

For working points sufficiently far from integer and halfinteger resonances, we have

$$
\frac{\beta_{\mathrm{ac}}}{2} \frac{K_{\mathrm{ac}}^{0}}{\tan \left(2 \pi Q_{\mathrm{nat}}^{0}\right)} \ll 1,
$$

and, considering the tune derivative versus intensity, we get

$$
\frac{\mathrm{d} K_{\mathrm{ac}}}{\mathrm{d} N_{b}}=-\frac{4 \pi}{\beta_{\mathrm{ac}}} \frac{\mathrm{d} Q_{\mathrm{nat}}}{\mathrm{d} N_{b}} .
$$

A step in the slope of phase advance given by Eq. (7) could be appreciated at the location of the ac dipole $s=s_{\mathrm{ac}}$

$$
\frac{\mathrm{d} \mu_{d}\left(s_{\mathrm{ac}}+\varepsilon\right)}{\mathrm{d} N_{b}}-\frac{\mathrm{d} \mu_{d}\left(s_{\mathrm{ac}}-\varepsilon\right)}{\mathrm{d} N_{b}}=-\frac{\mathrm{d} Q_{\mathrm{nat}}}{\mathrm{d} N_{b}} .
$$

with $\varepsilon \rightarrow 0$. Therefore, at the ac dipole location, a step corresponding to the opposite sum of all the impedanceinduced tune shifts would be observable.

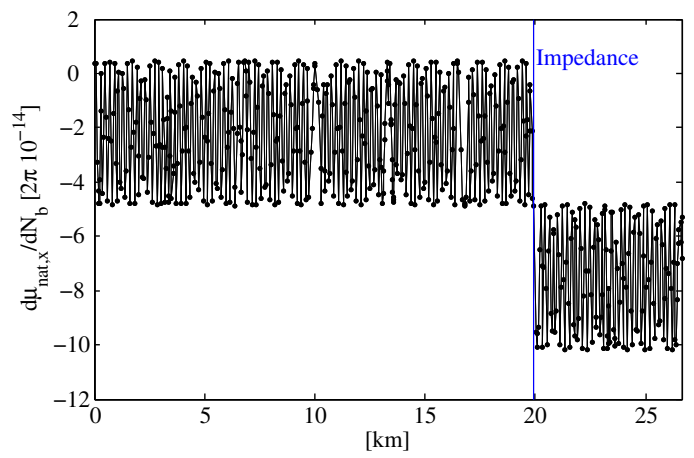

FIG. 3. Natural horizontal phase advance beating versus intensity calculated with HEADTAIL simulations of a broadband impedance placed close to the IP7 in the LHC at $20 \mathrm{~km}$. An horizontal kick is used as excitation.

This result is benchmarked with HEADTAIL. The LHC beam 1 ring is modeled including a broadband resonator in the IP7 collimation section. The tune shift is expected to be $\mathrm{d} Q_{\text {nat }} / \mathrm{d} N_{b} \simeq 5 \times 10^{-14}$. Simulations are performed with a kick and with an ac dipole excitation (as shown in Fig. 1). Figures 3 and 4 respectively show the phase advance measured at the natural tune and at the driven tune. In both cases the amplitude of the beating oscillation which is roughly the tune shift matches the expected value. Using an ac dipole as exciter, the step in the phase advance at the impedance location is compensated by another step at the ac dipole location as expected from Eq. (17).

This effect is intuitively explained by the fact that the driven ac dipole frequency does not change with intensity, and eventual steps in the phase advance slope versus intensity must be compensated at the ac dipole location.

Following these observations, a negative step in the phase advance variation with intensity is correlated with localized impedance sources, while a positive step at the ac dipole location is correlated with the opposite sum of all the machine impedances. An appropriate pseudoinversion $[6,9]$ can therefore be implemented associating defocusing quadrupoles to the impedances and a single focusing quadrupole in the ac dipole location.

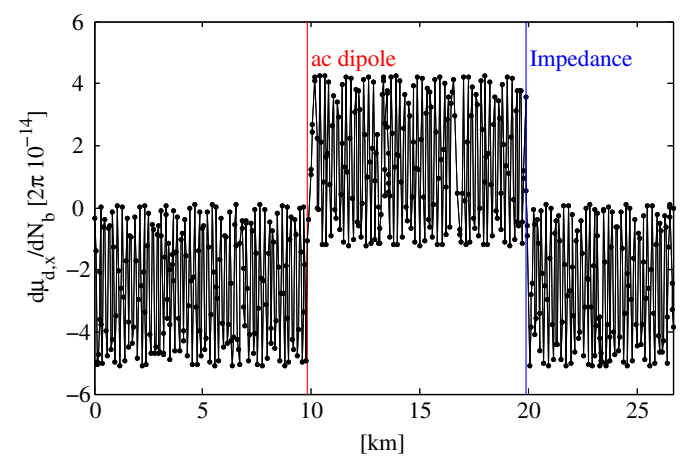

FIG. 4. Driven horizontal phase advance beating versus intensity calculated with HEADTAIL simulations of a broadband impedance placed close to the IP7 in the LHC at $20 \mathrm{~km}$. An ac dipole is used as excitation. 


\section{MEASUREMENT ACCURACY}

The accuracy of the method described in the previous section is mainly determined by the accuracy of the phase advance measurements. Estimates of the phase advance uncertainty are given below for different Fourier decomposition techniques [25].

\section{A. Uncertainty on phase advance}

Let us consider the following sinusoidal signals with same amplitude A, frequency $Q^{0}$ and phase advance $\Delta \mu=\mu_{2}-\mu_{1}$

$$
\begin{aligned}
& B P M_{1}(n)=\mathrm{A} \cos \left(2 \pi Q^{0} n+2 \pi \mu_{1}\right)+\mathrm{n}_{1}(n), \\
& B P M_{2}(n)=\mathrm{A} \cos \left(2 \pi Q^{0} n+2 \pi \mu_{2}\right)+\mathrm{n}_{2}(n),
\end{aligned}
$$

with $n \in\{0, \ldots, N-1\}, N$ the number of sampled turns, and $n_{1,2}(n)$ additive Gaussian noise of standard deviation $\sigma_{n}$. We define the noise to signal Ratio parameter as $\mathrm{NSR}=\sigma_{n} / \mathrm{A}$. Considering a number $\mathrm{M}$ of simulations, we can study how the noise impacts the uncertainty in tune and phase advance measurements. We define the uncertainty in tune determination $\sigma_{Q}$ as the standard deviation of the difference between measured and simulated tune frequencies over the $\mathrm{M}$ simulations, and analogously we define the phase advance uncertainty $\sigma_{\Delta \mu}$.

In the presence of noise, a classical fast Fourier transform (FFT) algorithm resolves $\sigma_{Q} \propto 1 / N$. Other accurate iterative methods like SUSSIX [26] or NAFF [27] give higher spectral resolution [28] up to $\sigma_{Q} \propto 1 / N^{3 / 2}$ (in the noise free case they get up to $\left.\sigma_{Q} \propto 1 / N^{4}\right)$. Figure 5 shows the tune uncertainty for increasing number of turns with a noise level of NSR $=5 \%$. Fitting the data we can get an estimation of the uncertainty as a function of the number of turns

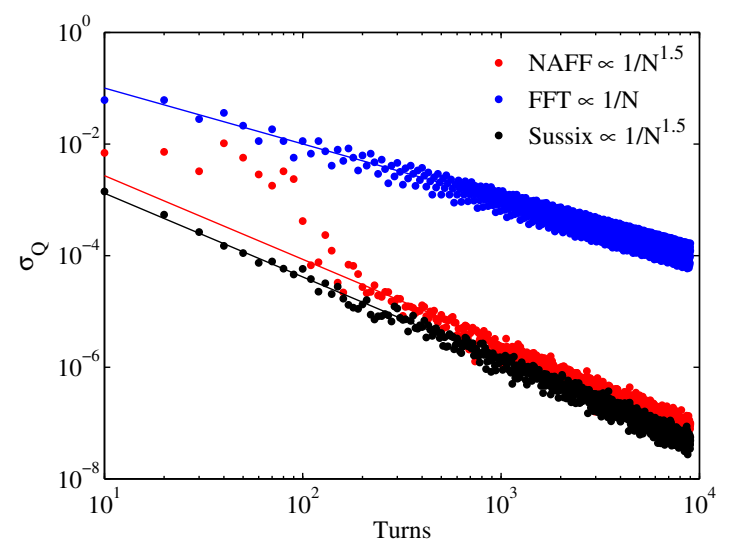

FIG. 5. Uncertainty in the tune determination with NAFF, FFT and SUSSIX versus number of turns calculated up to $N \simeq 10^{4}$ turns considering amplitude Gaussian noise of NSR $=5 \%$. Dots are the simulated data, lines are the fits. SUSSIX and NAFF both implement a hanning window.

$$
\sigma_{Q} \simeq F_{Q} \frac{\mathrm{NSR}}{N^{\alpha}},
$$

where $\alpha=1.5$ and $F_{Q} \simeq 0.82$ for SUSSIX, $\alpha=1.5$ and $F_{Q} \simeq 1.71$ for NAFF, $\alpha=1$ and $F_{Q} \simeq 20$ for the FFT.

Concerning the phase advance uncertainty, we can identify two possible correlated source of error: the direct impact of noise on the signal phase and the impact of noise through the window function.

The direct impact of noise on the signal phase was already studied in [29]. Defining the continuous Fourier transform (CFT) of a signal $x_{n}$ as

$$
X(Q)=\frac{1}{N} \sum_{n=1}^{N} \mathrm{e}^{-j 2 \pi Q(n-1)} x_{n}
$$

the spectrum $X_{1,2}$ corresponding to the sinusoidal signals of $B P M_{1,2}$ is given by

$X_{1,2}(Q)=\frac{\mathrm{A}}{2} \mathrm{e}^{j 2 \pi \mu_{1,2}} \mathcal{F}\left(Q-Q^{0}\right)+\frac{\mathrm{A}}{2} \mathrm{e}^{-j 2 \pi \mu_{1,2}} \mathcal{F}\left(Q+Q^{0}\right)$

where

$$
\mathcal{F}_{ \pm}(Q)=\frac{\sin \left(N \pi\left(Q \pm Q^{0}\right)\right)}{N \sin \left(\pi\left(Q \pm Q^{0}\right)\right)} \mathrm{e}^{-j\left(Q \pm Q^{0}\right)(N-1) / 2}
$$

is the CFT of the rectangular window implicitly enclosing the $\mathrm{N}$ signal samples.

An additive Gaussian noise $\mathrm{n}(n)$ introduces an uncertainty in the phase determination that can be quantified with the formula

$$
\sigma_{\mu} \simeq F_{\mu} \frac{\mathrm{NSR}}{\sqrt{N}},
$$

where $\sigma_{\mu}$ refers to the standard deviation of the measured phase of a single sinusoidal signal, and the numerical coefficient $F_{\mu}=\sqrt{2} / 2 \pi$. Considering the calculation of a phase advance between two signals, the error adds in quadrature

$$
\sigma_{\Delta \mu}^{2}=2 \sigma_{\mu}^{2},
$$

leading to

$$
\sigma_{\Delta \mu} \simeq F_{\Delta \mu} \frac{\mathrm{NSR}}{\sqrt{N}}
$$

with $F_{\Delta \mu}=1 / \pi$.

The impact of noise through the window function is significant only when measuring phase advances between two signals. Selecting the first part of Eq. (22), if the tune frequency at the two BPMs is different due to errors in the interpolation, there will be a contribution of the noise to the phase advance estimation through the phase of $\mathcal{F}$ given by

$$
\angle \mathcal{F}_{-}(Q)=-\left(Q-Q^{0}\right)(N-1) / 2 .
$$


For different measurements, the rms phase advance noise contribution is

$$
\sigma_{\angle \mathcal{F}}=\sigma_{Q}(N-1) / 2 .
$$

On the other hand, if the tune frequency at the two BPMs is the same, $\sigma_{\angle \mathcal{F}}=0$.

The contribution of noise on phase and through the window function are correlated with the noise signal. Therefore, we can estimate the total phase advance uncertainty as

$$
\sigma_{\Delta \mu}^{\mathrm{tot}}=\sigma_{\Delta \mu}+\sigma_{\angle \mathcal{F}}
$$

For interpolating methods like NAFF and SUSSIX we can write

$$
\sigma_{\Delta \mu}^{\mathrm{tot}}=F_{\Delta \mu}^{\mathrm{tot}} \frac{\mathrm{NSR}}{\sqrt{N}}
$$

with $F_{\Delta \mu}^{\text {tot }} \simeq F_{\Delta \mu}+F_{Q} / 2$.

For example, with SUSSIX we estimate $F_{\Delta \mu}^{\text {tot }} \simeq 1 / \pi+$ $0.82 / 2=0.73$ and for NAFF $F_{\Delta \mu}^{\text {tot }} \simeq 1.16$.

Figure 6 shows the comparison of FFT, SUSSIX and NAFF in phase advance uncertainty $\sigma_{\Delta \mu}$ over the number of turns. Fitting the data with Eq. (30) leaving $F_{\Delta \mu}^{\text {tot }}$ as a free parameter, we get in SUSSIX $F_{\Delta \mu}^{\text {tot }} \simeq 0.90$ and in NAFF $F_{\Delta \mu}^{\text {tot }} \simeq 1.30$ which are close to the expected values within a $20 \%$. The FFT can be fitted with $F_{\Delta \mu}^{\text {tot }} \simeq 0.37$ which is closer to the theoretical $F_{\Delta \mu}=1 / \pi$. This is not unexpected: SUSSIX and NAFF are interpolating methods while the FFT presents equally spaced spectrum samples fixed by the number of turns. The BPMs analyzed with an FFT will therefore exhibit the same frequency leading to $F_{\Delta \mu}^{\text {tot }} \simeq F_{\Delta \mu}=1 / \pi$.

\section{B. Uncertainty on phase advance slope with intensity}

Considering a set of $M$ phase advance measurements at different intensities $Y \in\left\{Y_{1}, Y_{2}, \ldots, Y_{M}\right\}$ with negligible

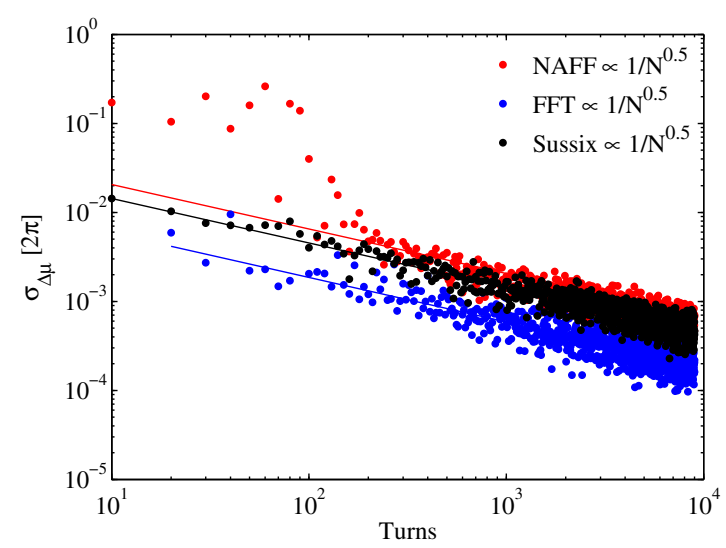

FIG. 6. Uncertainty in phase advance determination with NAFF, FFT and SUSSIX versus number of turns calculated up to $N \simeq 10^{4}$ turns considering amplitude Gaussian noise of $\mathrm{NSR}=5 \%$. Dots are the simulated data, lines are the fits. uncertainty in the intensity measurement, assuming the same uncertainty in phase advance for each measurement as given in Eq. (30), the uncertainty in the phase advance slope with intensity $\sigma_{\Delta \mu / \Delta N_{b}}$ will be given by [30]

$$
\sigma_{\Delta \mu / \Delta N_{b}}=F_{\Delta \mu}^{\mathrm{tot}} \frac{\mathrm{NSR}}{\sigma_{Y} \sqrt{M} \sqrt{N}},
$$

where $\sigma_{Y}$ is the standard deviation of the intensity scan $Y$. This uncertainty should be compared with the impedanceinduced phase advance amplitude of Eq. (7)

$$
A_{k}=\frac{\mathrm{d} Q_{y_{k}}}{\mathrm{~d} N_{b}} \frac{1}{\sin \left(2 \pi Q_{y}^{0}\right)} .
$$

\section{Estimates for the LHC}

The considerations done in the previous section suggest a method for measuring the total and localized impedance in circular accelerators with an ac dipole: the machine can be filled with a few well separated number of bunches with different intensities, the tune measured for the lowest intense one as a reference, the coherent oscillation excited with the ac dipole and the oscillations acquired for all the bunches at the same time. Alternatively, a single high intensity bunch could be scraped progressively in order to scan different intensities. From the transverse beam oscillation signal the driven optics can be inferred at each intensity. Taking the driven phase advance variation over the intensity scan we can access the information about the impedance distribution along the machine.

In this frame, a first exploratory measurement was done on 28-11-2012 in the LHC for the determination of the measurement uncertainty from multiturn data acquisition. A single high intensity bunch of $N_{b} \simeq 3 \times 10^{11}$ ppb was progressively scraped to $N_{b} \simeq 10^{11}$ ppb acquiring $M=14$ phase advance measurements at different intensities. Figure 7 shows the steps in the beam intensity caused

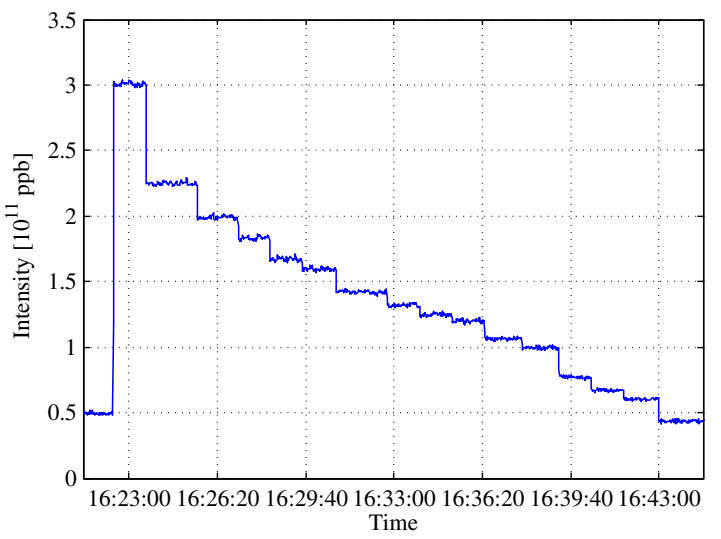

FIG. 7. Scraping of a single bunch during multiturn data acquisition with an ac dipole during the machine fill 3338 on 28-11-2012. 


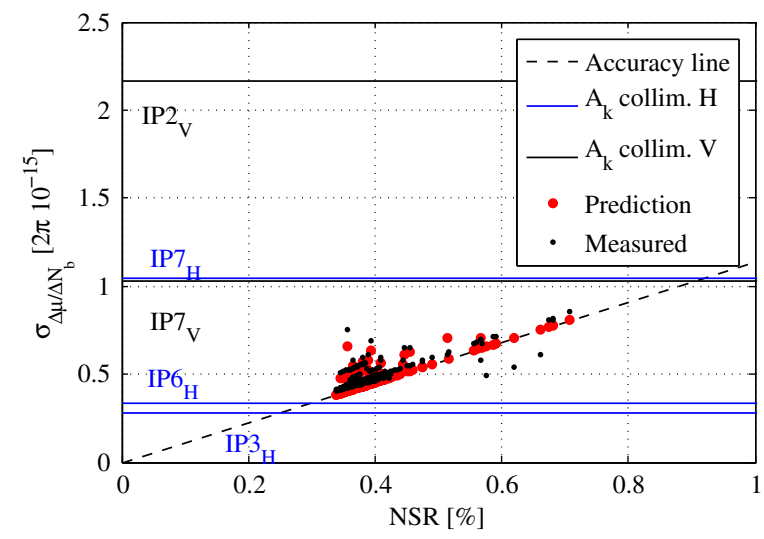

FIG. 8. Phase advance slope uncertainty in the LHC machine. Black dots represent the measured uncertainty for $N=2200$ turns and $M=14$ measurements over an intensity scan from $N_{b}=10^{11}$ to $N_{b}=3 \times 10^{11} \mathrm{ppb}$. Red dots represent the predicted uncertainty with Eq. (31). Horizontal lines are the signal amplitudes expected from the horizontal (in blue) and vertical (in black) collimators. The uncertainty line shows the uncertainty attainable over NSR for average measurement parameters.

by the scraping. The measurement was done at the injection energy $E=450 \mathrm{GeV}$ over 20 minutes. The machine natural tunes were $Q_{\text {nat, } y}^{0}=0.3085$ and $Q_{\text {nat }, x}^{0}=0.2743$ and the ac dipole oscillations were driven at $Q_{d, y}=0.32$ and $Q_{d, x}=0.27$. A number of $N=2200$ of coherent driven oscillations was recorded by the BPM system.

Figure 8 shows the uncertainty predicted with Eq. (31) and measured from the measured phase advances compared with the impedance-induced phase advance amplitudes of Eq. (32) of the most relevant collimator families at the interaction points (IPs). As we can see a good measurement accuracy is potentially achievable and the impedance of the collimators could, in principle, be localized in dedicated future measurements. Potentially better achievements could be obtained in Run 2 when a longer ac dipole plateau would be available [31]. Unfortunately this first measurement data did not show the expected behavior probably due to the parasitic LHC injection tune drift with time [32] which is in the order of $\Delta Q=5 \times 10^{-3}$ every $\sim 15$ minutes especially at injection. Since we are interested in measuring signals whose amplitude is one order of magnitude less, this effect shadows and could be reduced either waiting enough time before performing the measurements or measuring at top energy.

\section{CONCLUSIONS}

In this work we present analytical developments to interpret the impedance localization measurements performed by means of an ac dipole excitation together with uncertainly analysis.

The method is based on the acquisition of ac dipole induced betatron oscillations at the BPMs for different beam intensities. The observation of steps in the phase advance variation with intensity is associated with existing impedance sources: a negative step is correlated with localized impedance sources; a positive step at the ac dipole location is correlated with the opposite sum of all the machine impedances. An appropriate pseudoinversion can therefore be implemented associating defocusing quadrupoles to the impedances and a single focusing quadrupole in the ac dipole location.

In order to improve the measurement accuracy we compared different Fourier decomposition methods to calculate the phase advance between two sinusoidal signals in noisy environment. Given the same tune between two BPMs, the FFT algorithm is the most accurate in the phase advance detection, while, interpolating methods like NAFF and SUSSIX, introducing slightly different frequencies at the BPMs, increase the phase advance uncertainty.

For machines like the LHC, the higher accuracy reachable with an ac dipole with respect to the kick excitation represents an important argument in order to localize impedance sources. The first exploratory measurements in the LHC have been promising and a new campaign of impedance measurements is currently taking place.

This work is of particular importance for future projects like the FCC, in which the machine impedance will represent a problem [33] and accurate tools for impedance localization measurements are required.

\section{ACKNOWLEDGMENTS}

The authors would like to acknowledge B. Salvant and Y. Papaphilippou for the useful comments and suggestions received, R. Calaga, E. Métral, G. Papotti, F. Zimmermann and the LHC operation team for the support in the measurements performed in the LHC.

[1] F. J. Sacherer, Report No. CERN-MPS-INT-BR-74-8, 1974.

[2] D. Brandt, P. Castro, K. Cornelis, A. Hofmann, G. Morpurgo, G. L. Sabbi, J. Wenninger, and B. W. Zotter, Report No. CERN-SL-95-34 AP, 1995.

[3] V. Kiselev and V. Smaluk, in Proceedings of 4th European Workshop on Beam Diagnostics and Instrumentation for Particle Accelerators (DIPAC 99), edited by B. G. Martlew and R. J. Smith (Daresbury Lab., Daresbury, 1999), p. 202.

[4] L. Emery, G. Decker, and J. Galayda, in Proceedings of the Particle Accelerator Conference, Chicago, IL, 2001 (IEEE, New York, 2001), p. 1823.

[5] E. Plouviez and L. Farvacque, in Proceedings of the European Particle Accelerator Conference (EPAC), 2002, edited by J. L. Laclare (CERN, Geneva, 2002), p. 1550 .

[6] G. Arduini, C. Carli, and F. Zimmermann, Report No. CERN-AB-2004-083, 2004.

[7] R. Calaga, G. Arduini, E. Métral, G. Papotti, D. Quatraro, G. Rumolo, B. Salvant, and R. Tomás, Proceedings of the 23rd Particle Accelerator Conference, Vancouver, Canada, 2009 (IEEE, Piscataway, NJ, 2009). 
[8] R. Calaga, in APEX Workshop, Stony Brook University, NY, USA, 2010 (Stony Brook University, NY, 2010).

[9] N. Biancacci, Ph.D. thesis, University of Rome La Sapienza, 2014.

[10] R. Miyamoto, Diagnostics of the Fermilab Tevatron Using an AC Dipole (VDM Publishing, Saarbrücken, 2009).

[11] M. Bai et al., Overcoming Intrinsic Spin Resonances with an rf Dipole, Phys. Rev. Lett. 80, 4673 (1998).

[12] S. Peggs, in Proceedings of the 18th Particle Accelerator Conference, New York, 1999 (IEEE, New York, 1999), p. 1823.

[13] R. Tomás, Normal form of particle motion under the influence of an ac dipole, Phys. Rev. ST Accel. Beams 5, 054001 (2002).

[14] R. Miyamoto, S. E. Kopp, A. Jansson, and M. J. Syphers, Parametrization of the driven betatron oscillation, Phys. Rev. ST Accel. Beams 11, 084002 (2008).

[15] M. Bai, G. Wang, and L. Yang, in Proceedings of the International Particle Accelerator Conference, Kyoto, Japan (ICR, Kyoto, 2010).

[16] X. Shen, S. Lee, M. Bai, S. White, G. Robert-Demolaize, Y. Luo, A. Marusic, and R. Tomás, Application of independent component analysis to ac dipole based optics measurement and correction at the relativistic heavy ion collider, Phys. Rev. ST Accel. Beams 16, 111001 (2013).

[17] R. Tomás, T. Bach, R. Calaga, A. Langner, Y. Levinsen, E. Maclean, T. Persson, P. Skowronski, M. Strzelczyk, G. Vanbavinckhove, and R. Miyamoto, Record low $\beta$ beating in the LHC, Phys. Rev. ST Accel. Beams 15, 091001 (2012).

[18] F. Schmidt et al., CERN Technical Report No. AB-Note2003-031-MD, 2003.

[19] R. Tomás, M. Bai, R. Calaga, W. Fischer, A. Franchi, and G. Rumolo, Measurement of global and local resonance terms, Phys. Rev. ST Accel. Beams 8, 024001 (2005).

[20] S. White, E. Maclean, and R. Tomás, Direct amplitude detuning measurement with AC dipole, Phys. Rev. ST Accel. Beams 16, 071002 (2013).
[21] T. H. B. Persson, Y. Inntjore Levinsen, R. Tomás, and E. H. Maclean, Chromatic coupling correction in the large hadron collider, Phys. Rev. ST Accel. Beams 16, 081003 (2013).

[22] G. Rumolo and F. Zimmermann, CERN Technical Report No. SL-Note-2002-036-AP, 2002.

[23] N. Biancacci, HTWG meeting: HEADTAIL and MAD-X, CERN, Geneva, Switzerland, 2012.

[24] R. Tomás, Adiabaticity of the ramping process of an AC dipole, Phys. Rev. ST Accel. Beams 8, 024401 (2005).

[25] N. Biancacci, in The OMC Workshop (CERN, Geneva, 2013).

[26] R. Bartolini and F. Schmidt, in Proceedings of Nonlinear and Stochastic Beam Dynamics in Accelerators: A Challenge to Theoretical and Computational Physics, edited by A. Bazzani et al. (DESY, Hamburg, 1998), p. 390.

[27] J. Laskar, C. Froeschlé, and A. Celletti, The measure of chaos by the numerical analysis of the fundamental frequencies. Application to the standard mapping, Physica D Nonlinear Phenomena 56, 253 (1992).

[28] R. Bartolini, M. Giovannozzi, W. Scandale, A. Bazzani, and E. Todesco, Precise measurement of the betatron tune, Part. Accel. 55, 1 (1996).

[29] Y. Alexahin and E. Gianfelice-Wendt, Determination of linear optics functions from turn-by-turn data, J. Instrum. 6, P10006 (2011); Report No. FERMILAB-PUB-06-093$\mathrm{AD}, 2011$.

[30] R. J. Barlow, Statistics: A Guide to the Use of Statistical Methods in the Physical Sciences (Manchester Physics Series) (WileyBlackwell, New York, 1989).

[31] N. Magnin, in The OMC Workshop (CERN, Geneva, 2013).

[32] N. Aquilina, M. Lamont, R. Steinhagen, E. Todesco, J. Wenninger, and N. Sammut, Report No. CERN-ATS2012-196, 2012.

[33] X. Buffat and B. Salvant, Impedance Meeting: Introduction to FCC-hh Impedance (CERN, Geneva, Switzerland, 2015). 